\title{
P 039 WHOSE DECISION IS IT ANYWAY? PLANNING FUTURE CARE AT THE END OF LIFE FOR CARE HOME RESIDENTS
}

Tamsin McGlinchey, ${ }^{1}$ Philip Saltmarsh, ${ }^{1}$ Rebecca Bancroft, ${ }^{2}$ Stephen Mason, ${ }^{1}$

Maureen Gambles, ${ }^{1}$ Gerard Corcoran, ${ }^{1}$ John Ellershaw ${ }^{1} .{ }^{1}$ Marie Curie Palliative Care Institute Liverpool, Liverpool, UK; ${ }^{2}$ Royal Liverpool \& Broadgreen University Hospital NHS Trust, Liverpool, UK

\subsection{6/bmjspcare-2014-000654.80}

Background Advance Care Planning (ACP) is a process of discussion and shared decision making between an individual and their care provider. Discussion takes place in the context of an anticipated deterioration of the individual's condition. Shared decision making promoted by ACP has been highlighted as a key component in coordinating care at the end of life. This has particular significance for people who may lose mental capacity, enabling them to discuss, plan and document their wishes for possible future care.

Greater understanding of how ACP may support patients and their families is required. Accordingly, a study is currently engaged in the Care Home (CH) setting to assess the impact that ACP discussions have on all participants; residents, relatives/friends and healthcare professionals.

Aim(s) To examine; the timing of discussions around residents choices at the end of life and planning for future care the process of shared decision making in ACP.

Method(s) Interpretive phenomenology underpin the research methods employed; In-depth semi-structured interviews with $\mathrm{CH}$ residents (where possible), relatives/friends of residents and health care staff are being undertaken using a longitudinal case study design $(n=10)$ :

Initial interview after first ACP discussion

Follow up interview(s) at 3 monthly intervals throughout the study period

Post bereavement interview with relative/friends and healthcare professionals

Results Results to date show discussions about choices at the end of life and planning for future care are mostly happening between relatives and health care professionals due to a lack of mental capacity in care home residents at the time of ACP discussions.

Conclusion(s) More understanding is required, why discussions about choices at the end of life and planning for future care with residents in Care Homes are not taking place earlier in the residents disease trajectory and whilst they have capacity. 